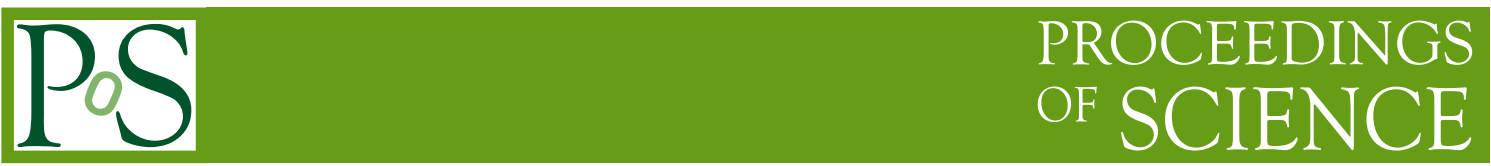

\title{
A new design for the CERN to Fréjus neutrino beam
}

\author{
E. Baussan, M. Dracos*, G. Gaudiot, B. Lepers, F. Osswald, P. Poussot, \\ N. Vassilopoulos, J. Wurtz and V. Zeter on behalf of EUROnu WP2 group \\ IPHC, Université de Strasbourg, CNRS/IN2P3 F-67037, Strasbourg, France \\ E-mail: marcos.dracoseiphc.cnrs.fr
}

\begin{abstract}
In the framework of the EUROnu design study, a new design for the CERN to Fréjus neutrino beam based on the SPL is under development by the WP2 group. This neutrino beam allows to significantly improve the measure on the mixing angle $\theta_{13}$ and $\mathrm{CP}$ violation by using a multi-MW proton beam of $4.5 \mathrm{GeV} / \mathrm{c}$, a baseline of $130 \mathrm{~km}$ and the future water Cherenkov MEMPHYS (440 kton fiducial mass) far detector. The main challenge of this project lies with the design of a multiMW target and hadronic collector for the proton beam. The horn and the decay tunnel parameters have been optimized with a novel procedure based on a scan of a multi-dimensional parameter space in order to maximize any potential discovery. The target design, thermo-mechanical analysis, and power supply design of the horn system as well as any safety issues are being studied to meet the MW power requirements of the proton beam. The results from the combined experiment of the Super-Beam and MEMPHYS detector and its high physics potential are also discussed.
\end{abstract}

The 2011 Europhysics Conference on High Energy Physics, EPS-HEP 2011,

July 21-27, 2011

Grenoble, Rhône-Alpes, France

${ }^{*}$ Speaker. 


\section{Physics potential}

The combined experiment of the Super Beam [1] with MEMPHYS detector [2] has an excellent discovery potential of $\sin ^{2} 2 \theta_{13}$ (extending to low values, figure 1 ) and CP-violation [3, 4]. Also, it has good sensitivity to the neutrino mass hierarchy when data from a $\beta$-beam and atmospheric neutrinos are combined [3]. The horn parameters as well as the geometrical parameters of the decay tunnel (length and radii) are optimized for the best achievable sensitivity limit on $\sin ^{2} 2 \theta_{13}$ $[4,5]$. The beam parameters are initially scanned broadly and then restrictly in three iterations in order to minimize the CP-violation averaged $99 \%$ C.L. high sensitivity limit on $\sin ^{2} 2 \theta_{13}$.

\section{The proton-beam and horn/target station}

A 4-MW proton-beam from CERN's SPL is foreseen to be separated by a series of kicker magnets into four beam lines. Then each beam will be focused by a series of quadruples and correctors to a four horn/target assembly (figure 2) [6]. In this way, each horn/target assembly would be able to accommodate better the multi-MW power and thus increasing its lifetime.

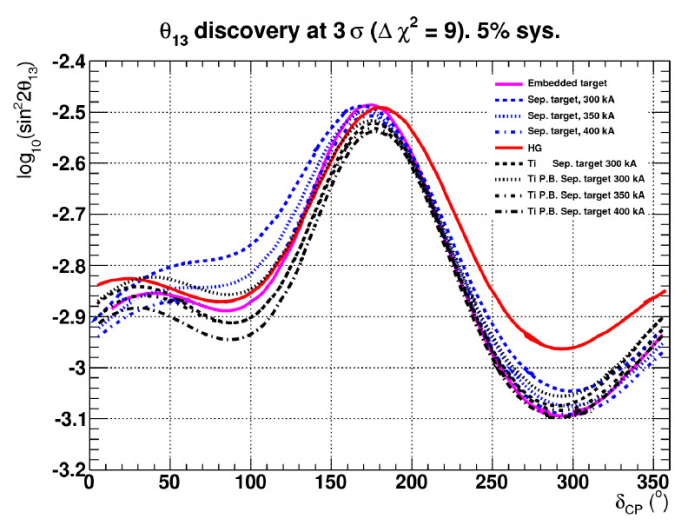

Figure 1: $\theta_{13}$ discovery limits.

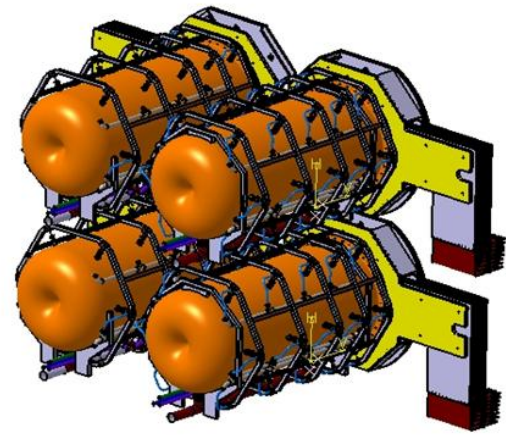

Figure 2: Four-horn assembly.

\section{Target and horn studies}

A packed-bed target with Ti6Al4V-spheres and helium transverse cooling has been chosen as the baseline target option [1]. It is placed inside the upstream part of horn's inner conductor. The advantages of this configuration are among others, large surface area for heat transfer with coolant able to access areas with highest energy deposition, minimal thermo-mechanical and inertial stresses and potential heat removal rates at the hundreds $\mathrm{kW}$ level with high helium flow rate.

Finite-element studies show that the aluminum alloy Al-6061T6 of the horn is subjected to cyclic deformation due to a pulsed magnetic pressure load. If a uniform temperature is achieved everywhere, the horn is expanding, and the maximum thermal static stress is $6 \mathrm{MPa}$ [7]. The combined stresses in the inner conductor due to the magnetic pulses and the thermal stress for a uniform achieved temperature of $60{ }^{\circ} \mathrm{C}$ are around $20 \mathrm{MPa}$. For the inner conductor horn, the 
magnetic pressure pulse creates a peak Von Mises stress of about $16 \mathrm{MPa}$ [7]. As result, preliminary fatigue studies for the horn show that the design is feasible for at least a year [7]. Finally, an onehalf sinusoid current waveform with a $350 \mathrm{kA}$ maximum current and pulse-length of $100 \mu \mathrm{s}$ at $12.5 \mathrm{~Hz}$ frequency is foreseen for each horn. In order to compensate for these extreme conditions, a power supply with energy recovery and 10 modules branched in parallel, with $35 \mathrm{kA}$ current each running at $50 \mathrm{~Hz}$ frequency is being studied.

\section{Safety}

The Super Beam facility is being designed to take into account the significant amount of radiation produced during beam operation and the radio-activation of the surrounding environment. The design of the shielding should reduce the dose equivalent rate to a minimal level. The Super Beam infrastructure will consist of: a) Proton Driver line, b) Experimental Hall (Target Station, Decay Tunnel, Beam Dump), c) Spare Area Room, d) Hot Cell, e) Service Galleries (Power supply, Cooling system) f) Air-Ventilation system and g) Waste Area.

\section{Conclusion}

Monte-Carlo studies show the high potential discovery of $\theta_{13}$ and CP-violation for the Super Beam, while thermo-mechanical and dynamical stress finite-element studies show that the fourhorn/target system can be feasible under the extreme $4 \mathrm{MW}$ proton-beam power.

\section{Acknowledgments}

We acknowledge the financial support of the European Community under the European Commission Framework Programme 7 Design Study: EUROnu, Project Number 212372. The EC is not liable for any use that may be made of the information contained herein.

\section{References}

[1] C. Bobeth et al, The target and horn for the SPL-based Super Beam: preliminary design report, [EUROnu-WP 2-11-01] (2011) http://www.euronu.org

[2] A. Tonazzo et al, The MEMPHYS project, Nucl.Instrum.Meth. A639 (2011) 287-289

[3] J.E. Campagne, M. Maltoni, M. Mezzetto, T. Schwetz Physics potential of the CERN-MEMPHYS neutrino oscillation project, [hep-ph/0603172v3]

[4] A. Longin, A new design for the CERN-Fréjus Super Beam, [EUROnu-WP2-10-04] (2010) http://www.euronu.org

[5] C. Bobeth and A Longhin, Optimization of hadron focusing for the SPL-Fréjus Super Beam, [EUROnu-WP2-10-02] (2010) http://www.euronu.org

[6] C. Bobeth et al, The proton driver for the neutrino Super Beam: status and issues, [EUROnu-WP2-09-11] (2009) http://www.euronu.org

[7] B. Lepers, Horn studies, (EUROnu-WP2-11-Xy, note to be submitted) (2011), Water jet cooling system for an electromagnetic horn, [EUROnu-WP 2-10-06] (2010) http://www.euronu.org 